\title{
Random walk and linear switching systems
}

\author{
Yulei Pang, Alex Wang, Xiaozhen Xue, and Clyde F. Martin
}

\begin{abstract}
In this paper we address the question "for a deck of cards, how many times a top-in shuffle should be performed before the top card goes back to the original position?" This problem has been studied in the literature but we are interested in the implications for linear switching systems. We simulate top-in shuffling for 6,12 , and 54 cards, and determine the underlying statistics. Finally we prove that the distribution of the stoping time is an exponential distribution, and the expect value approaches to that of the uniform distribution for large number of shuffling. We make essential use of the properties of linear, stochastic switching systems.
\end{abstract}

\section{Introduction}

Suppose we have a deck of $n$ cards, labeled by integers from 1 to $n$. We will number the deck so that an original unshuffled deck would be written $(1,2,3, \ldots, n)^{\prime}$. Hereafter, we will call this the natural order. From a mathematical viewpoint, shuffling a deck of $n$ cards can be thought of as a permutation of the the numbers from 1 to $n$. A deck of $n$ cards can be ordered in $n$ ! ways. The outcome order is dependent on which method we choose to shuffle the cards. A description of the most popular shuffles is described in [2]. In this paper we focus on just one type, the so called top-in shuffle. Top-in Shuffle: Take the card from the top and insert it at a random position in the deck [1].

An important part of this paper is to describe the random shuffling of a deck of cards as a linear switching system. We use some results from the theory of linear discrete time stochastic switching systems to describe limiting phenomena of shuffling.

In Section 2 we examine the dynamics of a repeated shuffle and the dynamics of a pair of interlacing shuffles. In Section 3 we introduce the idea of linear stochastic switching system as a model for shuffling. In Section 4 we examine the dynamics of the expected value of random shuffling using the theory of switching systems. In Section 5 We do an indepth study of top-in shuffling and report three simulations on various size decks. In Section 6 we 
construct the distribution of hitting time for all top in shuffles and show that each distribution is approximately geometric and hence has limit as an exponential distribution. The rate of convergence is explicit in the proof. In Section 7 we study the limiting behavior of the expected value of the top-in shuffle. Again the result is for decks of arbitrary size. Tables and code are contained in the appendices.

\section{Dynamics and control}

The basis for many tricks with cards revolve around the fact that if a deck is carefully shuffled using the same exact shuffle the cards will eventually return to their original order. We look at a series of simple examples before proving general results. One standard shuffle is given by the following example.

Let a deck of six cards be ordered from 1 to 6 , so we have a vector $(1,2,3,4,5,6)^{\prime}$. We shuffle by interlacing the two halves of the deck to obtain the ordering $(1,4,2,5,3,6)^{\prime}$. Repeating this we see that

$$
\left(\begin{array}{l}
1 \\
2 \\
3 \\
4 \\
5 \\
6
\end{array}\right) \rightarrow\left(\begin{array}{l}
1 \\
4 \\
2 \\
5 \\
3 \\
6
\end{array}\right) \rightarrow\left(\begin{array}{l}
1 \\
5 \\
4 \\
3 \\
2 \\
6
\end{array}\right) \rightarrow\left(\begin{array}{l}
1 \\
3 \\
5 \\
2 \\
4 \\
6
\end{array}\right) \rightarrow\left(\begin{array}{l}
1 \\
2 \\
3 \\
4 \\
5 \\
6
\end{array}\right)
$$

We can think of this as acting on the vector with the 6 by 6 matrix

$$
A=\left(\begin{array}{llllll}
1 & 0 & 0 & 0 & 0 & 0 \\
0 & 0 & 0 & 1 & 0 & 0 \\
0 & 1 & 0 & 0 & 0 & 0 \\
0 & 0 & 0 & 0 & 1 & 0 \\
0 & 0 & 1 & 0 & 0 & 0 \\
0 & 0 & 0 & 0 & 0 & 1
\end{array}\right)
$$

and the shuffling is represented by the dynamical system

$$
x_{n+1}=A x_{n} .
$$

The fact that it repeated is a simple consequence of the fact that $A^{4}=I$.

The underlying mathematics of this card trick is that the trickster appears to be randomizing the order of the deck by repeatedly shuffling but in fact he is simply rearranging the deck in a very precise manner so that in 
the end it is identical to the first position. The mathematics is simply that a shuffle is a permutation and the permutation can be represented by a single matrix. Because the permutation group is finite we must have that any matrix, $C$, that represents a shuffle, has to have the property that $C^{n}=I$ for some value of $\mathrm{n}$.

Now suppose we do really want to randomize the deck. As we see above a single permutation will not work. So let's consider another interlacing shuffle that is given by

$$
\left(\begin{array}{l}
1 \\
2 \\
3 \\
4 \\
5 \\
6
\end{array}\right) \rightarrow\left(\begin{array}{l}
4 \\
1 \\
5 \\
2 \\
6 \\
3
\end{array}\right) \rightarrow\left(\begin{array}{l}
2 \\
4 \\
6 \\
1 \\
3 \\
5
\end{array}\right) \rightarrow\left(\begin{array}{l}
1 \\
2 \\
3 \\
4 \\
5 \\
6
\end{array}\right)
$$

This quick calculation shows that the order of this permutation is 3 . The matrix representing this shuffle is given by

$$
D=\left(\begin{array}{llllll}
0 & 0 & 0 & 1 & 0 & 0 \\
1 & 0 & 0 & 0 & 0 & 0 \\
0 & 0 & 0 & 0 & 1 & 0 \\
0 & 1 & 0 & 0 & 0 & 0 \\
0 & 0 & 0 & 0 & 0 & 1 \\
0 & 0 & 1 & 0 & 0 & 0
\end{array}\right)
$$

If we simply alternate the two shuffles, we are just repeating the shuffle given by $A D$ and we know that $(A D)^{n}=I$ for some $n \leq 6$. But if we randomly choose the shuffle, we get a shuffle given by

$$
A^{n_{k}} D^{m_{k}} \cdots A^{n_{2}} D^{m_{2}} A^{n_{1}} D^{m_{1}},
$$

where $n_{i} \leq 3$ and $m_{i} \leq 2$. Now there are 6 ! possible shuffles and so it is a question of algebra if we can generate all the shuffles with these two. We will come back to this question later.

With a single shuffle we were able to represent it mathematically as the dynamical system $x_{n+1}=A x_{n}$, but here it is either represented as $x_{n+1}=$ $A x_{n}$ or randomly as $x_{n+1}=D x_{n}$. We can rewrite these two equations as one stochastic dynamic system

$$
x_{n+1}=(u(n) A+(1-u(n)) D) x_{n}
$$

where $u(n) \in\{0,1\}$ and $P\left(u_{n}=1\right)=.5$. 


\section{Switching systems}

A stochastic discrete time linear switching system is a stochastic system of the form

$$
x_{n+1}=\left(u_{1}(n) A_{1}+\cdots+u_{k}(n) A_{k}\right) x_{n}
$$

where the $u_{i}$ satisfy the following properties

$$
\begin{aligned}
u_{i}(n) & \in\{0,1\} \\
\sum_{i=1}^{k} u_{i}(n) & =1 .
\end{aligned}
$$

If the $u_{i}$ s are chosen randomly then we must assign a probability at each time $n$, i.e.

$$
\operatorname{Prob}\left(u_{i}(n)=1\right)=p_{i n}
$$

and for the purposes of this paper we will assume that the probabilities are constant with respect to $n$ and equal to $\frac{1}{n}$. See references [7] and [6] for examples and the treatment of such systems. An example of an application of a linear switching system is given in [5].

In this paper one of our goals is to describe a particular type of shuffle as a stochastic switching system. We use the top-in shuffle because of its simplicity but all shuffles can be represented by similar systems. Basically, any shuffle is given by a set of permutations acting on an ordered deck of cards. Thus the switching systems have as their natural state space the permutations of a single vector. Thus the matrices of the system in given by equation 3.1 are the elements of a matrix representation of the symmetric group. We can take the state space to be the set of $n$ ! vectors obtained by taking all permutations of the vector $(1,2, \ldots, n)^{\prime}$.

The questions usually ask about shuffles are somewhat foreign to systems theory but are interesting never the less. In systems theory the usual questions have to with stability, controllability, reachability, etc. We will, in the next section, study the expected value of these systems using material from [7].

\section{Some system theoretic questions}

In [7] it was shown that the expected value and the variance of a discrete time stochastic switching system could be computed recursively with associated 
dynamical systems. In [3] there is an indepth discussion of when a random walk on a finite group becomes uniformly distributed on the group elements. We examine this issue with the system of equation 2.1. From [7] we see that the expected value of the system acting on the space of permutations is given by

$$
E\left[x_{n+1}\right]=\frac{1}{2}(A+D) E\left[x_{n}\right]
$$

and

$$
F_{6}=\frac{1}{2}(A+D)=\frac{1}{2}\left(\begin{array}{cccccc}
1 & 0 & 0 & 1 & 0 & 0 \\
1 & 0 & 0 & 1 & 0 & 0 \\
0 & 1 & 0 & 0 & 1 & 0 \\
0 & 1 & 0 & 0 & 1 & 0 \\
0 & 0 & 1 & 0 & 0 & 1 \\
0 & 0 & 1 & 0 & 0 & 1
\end{array}\right)
$$

First it is clear that the kernel of $F_{6}$ is three dimensional and that the vector $(1,1,1,1,1,1)^{\prime}$ is an eigenvector with eigenvalue 1 . A bit of calculation yields that another eigenvector is given by $(1,1,-2,-2,1,1)^{\prime}$ with eigenvalue $-\frac{1}{2}$. With more calculation we find that $-\frac{1}{2}$ is an eigenvalue with multiplicity 2. The kernel of $F$ is spanned by the three vectors $(1,0,0,-1,0,0)^{\prime}$, $(0,1,0,0,-1,0)^{\prime}$ and $(0,0,1,0,0,-1)^{\prime}$. So it remains to find the generalized eigenvector associated with the eigenvalue $-\frac{1}{2}$. It is somewhat easier to calculate a vector that is orthogonal to the five known eigenvectors. It is easy to find $(-1,0,1,-1,0,1)^{\prime}$.

We now use this basis to represent the vector $(1,2,3,4,5,6)^{\prime}$. We write

$$
\begin{gathered}
x_{1}\left(\begin{array}{c}
1 \\
0 \\
0 \\
-1 \\
0 \\
0
\end{array}\right)+x_{2}\left(\begin{array}{c}
0 \\
1 \\
0 \\
0 \\
-1 \\
0
\end{array}\right)+x_{3}\left(\begin{array}{c}
0 \\
0 \\
1 \\
-0 \\
0 \\
-1
\end{array}\right)+x_{4}\left(\begin{array}{c}
1 \\
1 \\
-2 \\
-2 \\
1 \\
1
\end{array}\right) \\
+x_{5}\left(\begin{array}{l}
1 \\
1 \\
1 \\
1 \\
1 \\
1
\end{array}\right)+x_{6}\left(\begin{array}{c}
-1 \\
0 \\
1 \\
-1 \\
0 \\
1
\end{array}\right)=\left(\begin{array}{c}
1 \\
2 \\
3 \\
4 \\
5 \\
6
\end{array}\right) .
\end{gathered}
$$


We now act on both sides of this equation with the matrix $F_{6}$ to get

$$
x_{4}\left(\begin{array}{c}
-.5 \\
-.5 \\
1 \\
1 \\
.5 \\
.5
\end{array}\right)+x_{5}\left(\begin{array}{l}
1 \\
1 \\
1 \\
1 \\
1 \\
1
\end{array}\right)+x_{6}\left(\begin{array}{c}
-1 \\
-1 \\
0 \\
0 \\
1 \\
1
\end{array}\right)=\left(\begin{array}{l}
2.5 \\
2.5 \\
3.5 \\
3.5 \\
4.5 \\
4.5
\end{array}\right) .
$$

Some arithmetic shows that $x_{4}=0, x_{5}=3.5$ and $x_{6}=1$. Thus we have that

$$
\frac{1}{2}\left(\begin{array}{llllll}
1 & 0 & 0 & 1 & 0 & 0 \\
1 & 0 & 0 & 1 & 0 & 0 \\
0 & 1 & 0 & 0 & 1 & 0 \\
0 & 1 & 0 & 0 & 1 & 0 \\
0 & 0 & 1 & 0 & 0 & 1 \\
0 & 0 & 1 & 0 & 0 & 1
\end{array}\right)\left(\begin{array}{l}
1 \\
2 \\
3 \\
4 \\
5 \\
6
\end{array}\right)=3.5\left(\begin{array}{l}
1 \\
1 \\
1 \\
1 \\
1 \\
1
\end{array}\right)+\left(\begin{array}{c}
-1 \\
-1 \\
0 \\
0 \\
1 \\
1
\end{array}\right)
$$

After more calculation we that

$$
\lim _{n \rightarrow \infty} \frac{1}{2}\left(\begin{array}{cccccc}
1 & 0 & 0 & 1 & 0 & 0 \\
1 & 0 & 0 & 1 & 0 & 0 \\
0 & 1 & 0 & 0 & 1 & 0 \\
0 & 1 & 0 & 0 & 1 & 0 \\
0 & 0 & 1 & 0 & 0 & 1 \\
0 & 0 & 1 & 0 & 0 & 1
\end{array}\right)^{n}\left(\begin{array}{l}
1 \\
2 \\
3 \\
4 \\
5 \\
6
\end{array}\right)=3.5\left(\begin{array}{l}
1 \\
1 \\
1 \\
1 \\
1 \\
1
\end{array}\right) .
$$

We now calculate what the expected value of the uniform distribution would be. There are 5 ! vectors that have 1 in the first position. Thus the sum of all 6 ! vectors is given by $5 ! 6(6+1) / 2(1,1,1,1,1,1)^{\prime}$ and hence the expected value is $(3.5,3.5,3.5,3.5,3.5)^{\prime}$. Thus the expected value converges to the expected value of the uniform distribution. This does not guarantee that the system given by equation 2.1 results in a uniform distribution on the entire state space but it does not rule it out. So in order to determine if the system is reachable we still would have to do the algebra.

Another property of a stochastic system is the concept of hitting time. Many of the stopping time problems can be reduced to hitting times. In general what we are studying is the mixing of the deck and stopping when the deck is well mixed. An important treatment of mixing is given in [8] 
and [9]. Given an initial point and a large subset of the state space, we ask for the expected time for an orbit to intersect the subset. For the system we will consider in the next section, the subset is the set of all permutations with 1 as the first element. This set has cardinality $(n-1)$ ! and we will show that expected time to hit this set is governed by a geometric distribution and the limiting distribution as the deck size grows is exponential. On the other hand the question that we ask here is how long do we expect to operate before position one is filled by the first entry in the deck is an example of a strong uniform time.

\section{Experiment and analysis}

In this section, we will discuss the following questions:

(1) For a deck of cards, how many times of top-in shuffle should be performed before the top card goes back to the original position?

(2) To what distribution does the hitting time of the top-in shuffling conform?

We simulate top-in shuffling for 6 cards, 12 cards and extend the simulation to a standard deck of 54 cards, obtaining conjectures fore the distribution of the hitting time of the top-in shuffle.

\subsection{The top-in shuffling ${ }^{1}$}

If we consider shuffling 6 cards with top-in shuffle, using matrices to describe this process. A permutation can be represented by its incidence matrix.

$$
A_{1}=\left(\begin{array}{llllll}
1 & 0 & 0 & 0 & 0 & 0 \\
0 & 1 & 0 & 0 & 0 & 0 \\
0 & 0 & 1 & 0 & 0 & 0 \\
0 & 0 & 0 & 1 & 0 & 0 \\
0 & 0 & 0 & 0 & 1 & 0 \\
0 & 0 & 0 & 0 & 0 & 1
\end{array}\right) .
$$

If we take a card from the top and insert it at the $k$ th position in the deck, it corresponds to $A_{k}$, since in this example there are 5 other place

\footnotetext{
${ }^{1}$ Part of the remaining material is taken from [10].
} 
could be chosen for the top card. Hence, we will get the following matrices.

$$
\begin{aligned}
& A_{2}=\left(\begin{array}{cccccc}
0 & 1 & 0 & 0 & 0 & 0 \\
1 & 0 & 0 & 0 & 0 & 0 \\
0 & 0 & 1 & 0 & 0 & 0 \\
0 & 0 & 0 & 1 & 0 & 0 \\
0 & 0 & 0 & 0 & 1 & 0 \\
0 & 0 & 0 & 0 & 0 & 1
\end{array}\right) \\
& A_{3}=\left(\begin{array}{llllll}
0 & 1 & 0 & 0 & 0 & 0 \\
0 & 0 & 1 & 0 & 0 & 0 \\
1 & 0 & 0 & 0 & 0 & 0 \\
0 & 0 & 0 & 1 & 0 & 0 \\
0 & 0 & 0 & 0 & 1 & 0 \\
0 & 0 & 0 & 0 & 0 & 1
\end{array}\right), \quad A_{4}=\left(\begin{array}{llllll}
0 & 1 & 0 & 0 & 0 & 0 \\
0 & 0 & 1 & 0 & 0 & 0 \\
0 & 0 & 0 & 1 & 0 & 0 \\
1 & 0 & 0 & 0 & 0 & 0 \\
0 & 0 & 0 & 0 & 1 & 0 \\
0 & 0 & 0 & 0 & 0 & 1
\end{array}\right), \\
& A_{5}=\left(\begin{array}{llllll}
0 & 1 & 0 & 0 & 0 & 0 \\
0 & 0 & 1 & 0 & 0 & 0 \\
0 & 0 & 0 & 1 & 0 & 0 \\
0 & 0 & 0 & 0 & 1 & 0 \\
1 & 0 & 0 & 0 & 0 & 0 \\
0 & 0 & 0 & 0 & 0 & 1
\end{array}\right), A_{6}=\left(\begin{array}{llllll}
0 & 1 & 0 & 0 & 0 & 0 \\
0 & 0 & 1 & 0 & 0 & 0 \\
0 & 0 & 0 & 1 & 0 & 0 \\
0 & 0 & 0 & 0 & 1 & 0 \\
0 & 0 & 0 & 0 & 0 & 1 \\
1 & 0 & 0 & 0 & 0 & 0
\end{array}\right) .
\end{aligned}
$$

For example, if we insert the top card in the 4th position, it corresponds to the matrix $A_{4}$ above.

We assume that the cards are represented by an incidence vector with initial vector

$$
X_{0}=(1,2,3,4,5,6)^{\prime}
$$

and then the act of shuffling can be represented in the form

$$
X_{1}=A_{i} X_{0}
$$

where the $A_{i}$ s are as above.

After determining the incidence matrices for top-in shuffle of 6 cards, now consider shuffling two or even more times consecutively, which can be represented as a random product of the 6 matrices, i.e. $X_{2}=A_{j} A_{i} X_{0}$. Note that since the 6 matrices are from a group the $X_{i}$ is simply a permutation of the ordering in $X_{0}$. The question that we will answer and examine in this paper is what is the expected length of the random product until a vector of the form $X=\left(1, i_{2}, \ldots, i_{n}\right)^{\prime}$ is achieved, i.e. how long does it take for the top card to return to the top. 
If we use $X_{n}$ to denote the corresponding vector after the $n$th shuffle then we will get the relationship between $X_{n+1}$ and $X_{n}$ as

$$
X_{n+1}=\left(\delta_{1}(n) A_{1}+\delta_{2}(n) A_{2}+\cdots+\delta_{6}(n) A_{6}\right) X_{n}
$$

with

$$
\begin{aligned}
\delta_{i}(n) & \in\{0,1\} \\
\sum_{i=1}^{6} \delta_{i}(n) & =1 \\
P\left(\delta_{i}(n)=1\right) & =1 / 6
\end{aligned}
$$

That is the process can be represented as a linear stochastic switching system.

\subsection{Simulation}

In this section, we still use 6,12 and 54 cards as examples, Using 6 cards and doing complete shuffles 1000 times, we get Table (a). The data sets have been moved to the appendix. The odd numbered column in the table (a) represents the number of shuffles needed to move the top card back to the top position. The even column stands for how many times this situation will happen in the 1000 complete shuffles. For example, if we look at the line $(6,54 \mid 31,0)$ which means in the 1000 complete shuffles there are 54 times after we took 6 shuffles to bring the top card back to the top and 0 times after 31 shuffles. Meanwhile we realize with the growing first column, the second column contains more and more 0s. As a result, truncating insignificant trailing zeroes we can plot the histogram in Figure 1 and calculate the mean and standard deviation for this data set. The $\mathrm{C}++$ code for 6 cards is contained in the appendix.

We repeat the above process for 12 cards to get the data in Table (b) and shuffle 10,000 times to obtain the histogram in Figure 2. Similarly, we also extend it to the entire standard deck of cards, (52 cards plus two jokers) for which we need many more shuffles to get the approximate distribution. So we do the top-in shuffling 100,000 times (see Table (c) and obtain histogram graph (see Figure 3).

The statistics from these experiments and the figures, suggest that the number of shuffles to return 1 to the top spot satisfies the exponential distribution. In the following we will prove this. 


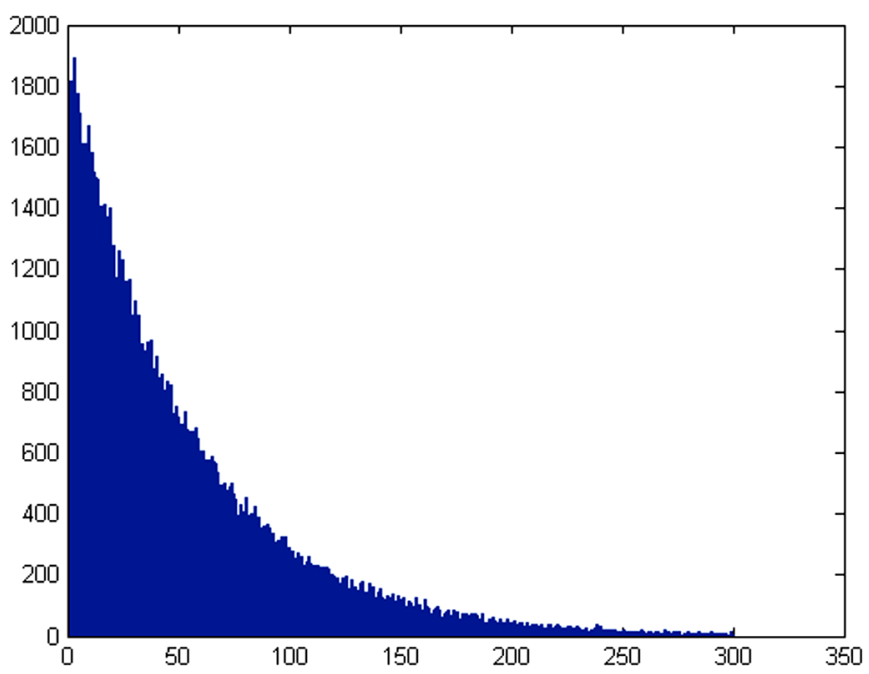

Figure 1: Steps needed for shuffling 6 cards when the top card goes back to original position. Mean $=6.02$, Standard deviation: 5.55 .

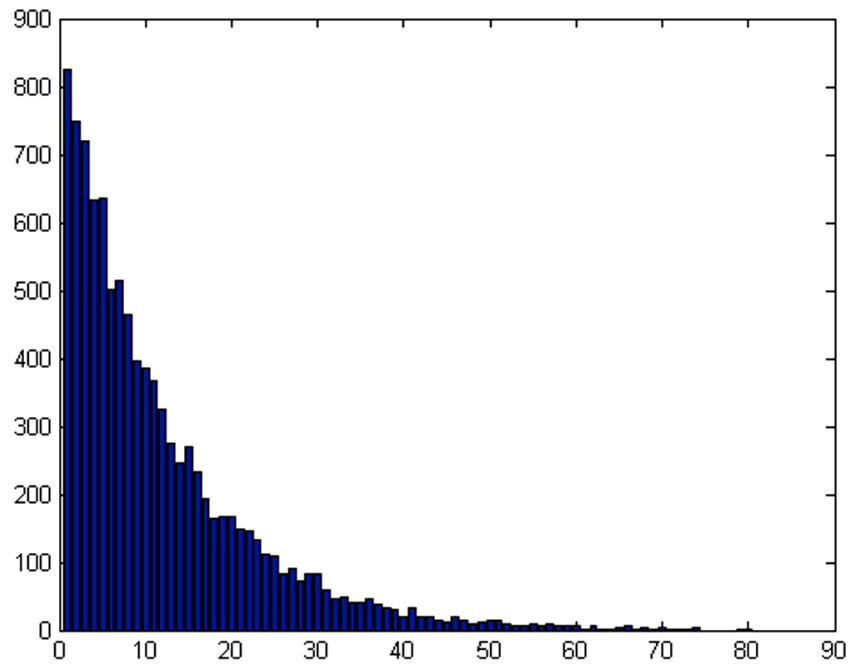

Figure 2: Steps needed for shuffling 12 cards when the top card goes back to original position. Mean; 11.97, Standard deviation; 11.48.

\section{Exponential distribution}

In this section, we prove some results about the distribution of the number of shuffles to make the top card back to top again. These results are indicated strongly by our experiments. 


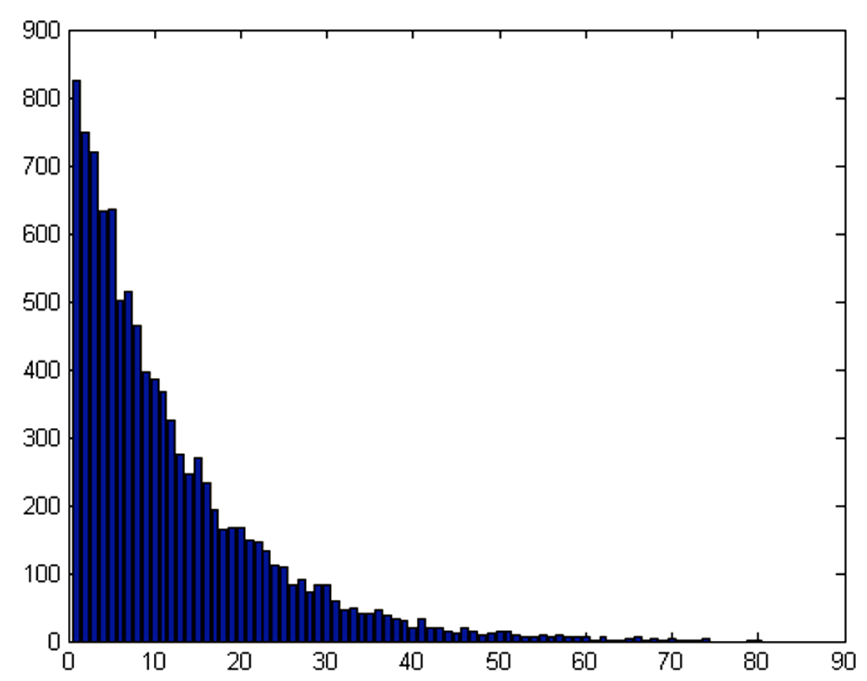

Figure 3: Steps needed for shuffling 54 cards when the top card goes back to original position. Mean $=53.93$, Standard deviation $=53.47$.

Theorem 6.1. Let $x$ be the number of shuffles to make the top card back to top again the first time. For a deck of $k$ cards, the distribution of $x$ is

$$
p(x)= \begin{cases}\frac{1}{k}\left(\frac{k-1}{k}\right)^{x-1}, & x \geq 1 \\ 0, & x<0 .\end{cases}
$$

Proof. Clearly $x \geq 1$, so the distribution is 0 for $x<1$.

Let $x_{i}$ be the position of the original top card after $i$ shuffles before it returns back to the top. We claim that

$$
P\left(x_{i}=j\right)=\frac{1}{k}\left(\frac{k-1}{k}\right)^{i-1} \quad \text { for all } j=2,3, \ldots, k .
$$

We use induction on $i$. It is true for $i=1$ because the probability of top card going to any position after one shuffle is $1 / k$. Assume it is true for $i$. Then

$$
P\left(x_{i+1}=j\right)=\sum_{m=1}^{k} P\left(x_{i+1}=j \mid x_{i}=m\right) P\left(x_{i}=m\right) .
$$

Notice that

$$
P\left(x_{i+1}=j \mid x_{i}=m\right)=0 \quad \text { for all } m \neq j, j+1,
$$


and since there are $j-1$ positions above the $j$ th card and $k-j+1$ positions below the $j$ th card, we have

$$
\begin{aligned}
& P\left(x_{i+1}=j \mid x_{i}=j\right)=\frac{j-1}{k} \text { and } \\
& P\left(x_{i+1}=j \mid x_{i}=j+1\right)=\frac{k-(j+1)+1}{k}=\frac{k-j}{k} .
\end{aligned}
$$

So

$$
\begin{aligned}
P\left(x_{i+1}=j\right)= & P\left(x_{i+1}=j \mid x_{i}=j\right) P\left(x_{i}=j\right) \\
& +P\left(x_{i+1}=j \mid x_{i}=j+1\right) P\left(x_{i}=j+1\right) \\
= & \frac{j-1}{k}\left(\frac{1}{k}\right)\left(\frac{k-1}{k}\right)^{i-1}+\frac{k-j}{k}\left(\frac{1}{k}\right)\left(\frac{k-1}{k}\right)^{i-1} \\
= & \frac{1}{k}\left(\frac{k-1}{k}\right)^{i}, \quad \text { for all } j=2, \ldots, k-1
\end{aligned}
$$

and

$$
\begin{aligned}
P\left(x_{i+1}=k\right) & =P\left(x_{i+1}=k \mid x_{i}=k\right) P\left(x_{i}=k\right) \\
& =\frac{k-1}{k}\left(\frac{1}{k}\right)\left(\frac{k-1}{k}\right)^{i-1}=\frac{1}{k}\left(\frac{k-1}{k}\right)^{i} .
\end{aligned}
$$

Now we are ready to prove the result. Clearly for $j=1$

$$
p(1)=P(x=1)=\frac{1}{k} .
$$

For every $j \geq 2$

$$
\begin{aligned}
p(j)=P(x=j) & =P\left(x=j \mid x_{j-1}=2\right) P\left(x_{j-1}=2\right) \\
& =\left(\frac{k-1}{k}\right)\left(\frac{1}{k}\left(\frac{k-1}{k}\right)^{j-2}\right) \\
& =\frac{1}{k}\left(\frac{k-1}{k}\right)^{j-1} \\
& =\frac{1}{k}\left(\frac{k-1}{k}\right)^{x-1} .
\end{aligned}
$$

Theorem 6.2. Let $x$ be the number of shuffles to make the top card returns to the top again the first time. Then for a deck of $k$ cards, 
i) the mean of $x$ is $E(x)=k$;

ii) the $m$-th moment $E\left(x^{m}\right)$ satisfies

$$
E\left(x^{m}\right)=k^{2} \frac{d}{d k}\left(\frac{k-1}{k} E\left(x^{m-1}\right)\right)+(k-1) E\left(x^{m-1}\right)
$$

and

$$
E\left(x^{m}\right)=m ! k^{m}+O\left(k^{m-1}\right) ;
$$

iii) the standard deviation of $x$ is $\sigma=\sqrt{k^{2}-k}$.

Proof. $\quad$ i) The mean of $x$ is given by

$$
\begin{aligned}
E(x) & =\sum_{i=1}^{\infty} i p(i)=\sum_{i=1}^{\infty} \frac{i}{k}\left(\frac{k-1}{k}\right)^{i-1} \\
& =k \frac{d}{d k} \sum_{i=1}^{\infty}\left(\frac{k-1}{k}\right)^{i}=k \frac{d}{d k}(k-1)=k .
\end{aligned}
$$

ii)

$$
\begin{aligned}
& k^{2} \frac{d}{d k}\left(\frac{k-1}{k} E\left(x^{m-1}\right)\right)+(k-1) E\left(x^{m-1}\right) \\
= & k^{2} \frac{d}{d k} \sum_{i=1}^{\infty} \frac{i^{m-1}}{k}\left(1-\frac{1}{k}\right)^{i}+(k-1) \sum_{i=1}^{\infty} \frac{i^{m-1}}{k}\left(1-\frac{1}{k}\right)^{i-1} \\
= & k^{2}\left(\sum_{i=1}^{\infty} \frac{-i^{m-1}}{k^{2}}\left(1-\frac{1}{k}\right)^{i}+\sum_{i=1}^{\infty} \frac{i^{m}}{k^{3}}\left(1-\frac{1}{k}\right)^{i-1}\right) \\
& +(k-1) \sum_{i=1}^{\infty} \frac{i^{m-1}}{k}\left(1-\frac{1}{k}\right)^{i-1} \\
= & \sum_{i=1}^{\infty} \frac{-i^{m-1}(k-1)}{k}\left(1-\frac{1}{k}\right)^{i-1}+\sum_{i=1}^{\infty} \frac{i^{m}}{k}\left(1-\frac{1}{k}\right)^{i-1} \\
& +(k-1) \sum_{i=1}^{\infty} \frac{i^{m-1}}{k}\left(1-\frac{1}{k}\right)^{i-1} \\
= & \sum_{i=1}^{\infty} \frac{i^{m}}{k}\left(1-\frac{1}{k}\right)^{i-1}=E\left(x^{m}\right) \text { for } m=2,3, \ldots
\end{aligned}
$$


To prove $E\left(x^{m}\right)=m ! k^{m}+O\left(k^{m-1}\right)$, we use induction on $m$. It is true for $m=1$. Assume it is true for $m-1, E\left(x^{m-1}\right)=(m-1) ! k^{m-1}+$ $O\left(k^{m-2}\right)$, then

$$
\begin{aligned}
E\left(x^{m}\right)= & k^{2} \frac{d}{d k}\left((m-1) ! k^{m-1}+O\left(k^{m-2}\right)\right) \\
& +(k-1)\left((m-1) ! k^{m-1}+O\left(k^{m-2}\right)\right) \\
= & k^{2}\left((m-1)(m-1) ! k^{m-2}+O\left(k^{m-3}\right)\right) \\
& +(m-1) ! k^{m}+O\left(k^{m-1}\right) \\
= & (m-1)(m-1) ! k^{m}+(m-1) ! k^{m}+O\left(k^{m-1}\right) \\
= & m ! k^{m}+O\left(k^{m-1}\right) .
\end{aligned}
$$

iii) By ii)

$$
E\left(x^{2}\right)=k^{2} \frac{d}{d k}\left(\frac{k-1}{k} k\right)+(k-1) k=2 k^{2}-k,
$$

and

$$
\sigma=\sqrt{E\left(x^{2}\right)-E(x)^{2}}=\sqrt{k^{2}-k}
$$

Theorem 6.3. As the number of cards $k$ becomes large, the distribution $p(x)$ asymptotically approaches to exponential distribution that

$$
f(x)= \begin{cases}\frac{1}{k} \exp \left(-\frac{1}{k}(x-1)\right), & x \geq 1 \\ 0, & x<1\end{cases}
$$

for each fixed $x$.

Proof. Since

$$
p(x)= \begin{cases}\frac{1}{k}\left(\frac{k-1}{k}\right)^{x-1}, & x \geq 1 \\ 0, & x<0\end{cases}
$$

Note that

$$
\frac{1}{k}\left(\frac{k-1}{k}\right)^{x-1}=\frac{1}{k} \exp \left((x-1) \log \left(1-\frac{1}{k}\right)\right)
$$


Therefore

$$
p(x)= \begin{cases}\frac{1}{k} \exp \left((x-1) \log \left(1-\frac{1}{k}\right)\right), & x \geq 1 \\ 0, & x<0 .\end{cases}
$$

Since

$$
\log \left(1-\frac{1}{k}\right)=-\frac{1}{k}+O\left(\frac{1}{k^{2}}\right)
$$

So for each fixed $x, p(x)$ approaches to

$$
f(x)= \begin{cases}\frac{1}{k} \exp \left(-\frac{1}{k}(x-1)\right), & x \geq 1 \\ 0, & x<1\end{cases}
$$

as $k$ becomes large.

\section{Distribution}

We begin by calculating the expected value for shuffling of the 6 card deck in the example. The expected value is given by the

$$
E\left[x_{n+1}\right]=F E\left[x_{n}\right]
$$

where $F$ is given by the weighted sum of the matrices.

$$
F=\frac{1}{6}\left(\begin{array}{cccccc}
1 & 5 & 0 & 0 & 0 & 0 \\
1 & 1 & 4 & 0 & 0 & 0 \\
1 & 0 & 2 & 3 & 0 & 0 \\
1 & 0 & 0 & 3 & 2 & 0 \\
1 & 0 & 0 & 0 & 4 & 1 \\
1 & 0 & 0 & 0 & 0 & 5
\end{array}\right)
$$

It is easy to see that there are eigenvalues $1, \frac{2}{3}$ and 0 but it is not so obvious that there are eigenvalues $\frac{1}{6}, \frac{1}{3}$ and $\frac{1}{2}$. These are discovered by taking advantage of the structure of $F$ which make the characteristic polynomial fairly easy to evaluate. Since there is a dominate eigenvalue the system will converge to the final distribution. It is best to start with the unit vector $(1,2,3,4,5,6)^{\prime}$. If we had the eigenvectors of $F$ then the we could expand the initial vector in terms of the eigenvectors and the limit would be given by the coefficient of the eigenvector $(1,1,1,1,1,1)$. The structure of the eigenvectors is not immediately obvious. 


$$
\begin{aligned}
& F_{6}^{10}\left[\begin{array}{l}
1 \\
2 \\
3 \\
4 \\
5 \\
6
\end{array}\right]=\left[\begin{array}{l}
3.4566 \\
3.4740 \\
3.4913 \\
3.5087 \\
3.5260 \\
3.5434
\end{array}\right], F_{6}^{20}\left[\begin{array}{l}
1 \\
2 \\
3 \\
4 \\
5 \\
6
\end{array}\right]=\left[\begin{array}{l}
3.4992 \\
3.4995 \\
3.4998 \\
3.5002 \\
3.5005 \\
3.5008
\end{array}\right], \text { and } \\
& F_{6}^{30}\left[\begin{array}{l}
1 \\
2 \\
3 \\
5 \\
6
\end{array}\right]=\left[\begin{array}{l}
3.5000 \\
3.5000 \\
3.5000 \\
3.5000 \\
3.5000 \\
3.5000
\end{array}\right] .
\end{aligned}
$$

Generally for a deck of $n$ cards we have the following results:

Theorem 7.1. The eigenpairs of $F$ are

$$
\left(1, \beta_{n}\right), \quad\left(0,\left[\begin{array}{c}
-n+1 \\
\beta_{n-1}
\end{array}\right]\right), \quad\left(\frac{k}{n},\left[\begin{array}{c}
k-n+1 \\
\alpha_{k} \\
\beta_{n-k-1}
\end{array}\right]\right) \quad \text { for } k=1, \ldots, n-2
$$

where $\beta_{i}$ is the $i$-vector with all entries equal 1 and

$$
\begin{aligned}
\alpha_{k} & =\left[\begin{array}{c}
a_{k 1} \\
\vdots \\
a_{k k}
\end{array}\right] \quad \text { with } a_{k 1}=\frac{(1-k)(n-k-1)}{n-1} \text { and } \\
a_{k i} & =\frac{n-k-1+(k+1-i) a_{i-1}}{n-i}, \text { for } i=2, \ldots, k .
\end{aligned}
$$

Proof. The eigenvectors associated with eigenvalues 1 and 0 are obvious. For each $k=1, \ldots, n-2$, write

$$
F=\frac{1}{n}\left[\begin{array}{cccccc}
1 & n-1 & 0 & \cdots & \cdots & 0 \\
1 & 1 & n-2 & \ddots & & \vdots \\
\vdots & 0 & 2 & \ddots & \ddots & \vdots \\
\vdots & \vdots & \ddots & \ddots & 2 & 0 \\
\vdots & \vdots & & \ddots & n-2 & 1 \\
1 & 0 & \ldots & \cdots & 0 & n-1
\end{array}\right]=\frac{1}{n}\left[\begin{array}{ccc}
\beta_{k} & A_{k} & 0 \\
1 & \gamma_{k} & \delta_{k} \\
\beta_{n-k-1} & 0 & B_{k}
\end{array}\right]
$$


where $A_{k}, B_{k}, \gamma_{k}, \delta_{k}$ are $k \times k,(n-k-1) \times(n-k-1), 1 \times k, 1 \times(n-k-1)$ matrices, respectively, given by

$A_{k}=\left[\begin{array}{cccc}n-1 & 0 & \cdots & 0 \\ 1 & \ddots & \ddots & \vdots \\ \vdots & \ddots & \ddots & 0 \\ 0 & \cdots & k-1 & n-k\end{array}\right], B_{k}=\left[\begin{array}{cccc}k+1 & n-k-2 & \cdots & 0 \\ 0 & \ddots & \ddots & \vdots \\ \vdots & \ddots & \ddots & 1 \\ 0 & \cdots & 0 & n-1\end{array}\right]$, $\gamma_{k}=[0, \ldots, 0, k], \quad \delta_{k}=[n-k-1,0, \ldots, 0]$.

Let

$$
\frac{1}{n}\left[\begin{array}{ccc}
\beta_{k} & A_{k} & 0 \\
1 & \gamma_{k} & \delta_{k} \\
\beta_{n-k-1} & 0 & B_{k}
\end{array}\right]\left[\begin{array}{c}
k-n+1 \\
\alpha_{k} \\
\beta_{n-k-1}
\end{array}\right]=\frac{k}{n}\left[\begin{array}{c}
k-n+1 \\
\alpha_{k} \\
\beta_{n-k-1}
\end{array}\right]
$$

we then have

$$
\begin{gathered}
(k-n+1) \beta_{k}+A_{k} \alpha_{k}=k\left((k-n+1) e_{1}+E_{k} \alpha_{k}\right), \\
e_{1}=\left[\begin{array}{c}
1 \\
0 \\
\vdots \\
0
\end{array}\right], E_{k}=\left[\begin{array}{cccc}
0 & \cdots & \cdots & 0 \\
1 & \ddots & & \vdots \\
\vdots & \ddots & \ddots & \vdots \\
0 & \cdots & 1 & 0
\end{array}\right] \\
k-n+1+\gamma_{k} \alpha_{k}+\delta_{k} \beta_{n-k-1}=k a_{k k}, \\
(k-n+1) \beta_{n-k-1}+B_{k} \beta_{n-k-1}=k \beta_{n-k-1} .
\end{gathered}
$$

(7.4) is clearly satisfied because $\left(n-1, \beta_{n-k-1}\right)$ is an eigenpair of $B_{k}$.

(7.3) is an identity

$$
k-n+1+k a_{k k}+n-k-1=k a_{k k} .
$$

(7.2) is equivalent to $\left(A_{k}-k E_{k}\right) \alpha_{k}=-k(n-k-1) e_{1}+(n-k-1) \beta_{k}$, or

$$
\left[\begin{array}{cccc}
n-1 & 0 & \cdots & 0 \\
-k+1 & \ddots & \ddots & \vdots \\
\vdots & \ddots & \ddots & 0 \\
0 & \cdots & -1 & n-k
\end{array}\right] \alpha_{k}=\left[\begin{array}{c}
(1-k)(n-k-1) \\
n-k-1 \\
\vdots \\
n-k-1
\end{array}\right]
$$

The iteration formula for the entries of $\alpha_{k}$ follows immediately. 
Theorem 7.2. The expected value $E\left[x_{m}\right]$ approaches to that of the uniform distribution as $m \rightarrow \infty$, i.e.

$$
\lim _{m \rightarrow \infty} F^{m}\left[\begin{array}{c}
1 \\
\vdots \\
n
\end{array}\right]=\frac{n+1}{2}\left[\begin{array}{c}
1 \\
\vdots \\
1
\end{array}\right] .
$$

Proof. Let $v_{k}$ be the eigenvector of $F$ associated with the eigenvalue $k / n$, $k=0,1, \ldots, n-2, n$, and let $x=[1,2, \ldots, n]^{\prime}$. Then $x=c_{0} v_{0}+\cdots+$ $c_{n-2} v_{n-2}+c_{n} v_{n}$ for some $c_{0}, \ldots, c_{n-2}, c_{n}$ and

$\lim _{m \rightarrow \infty} F^{n} x=\lim _{m \rightarrow \infty}\left(c_{1}\left(\frac{1}{n}\right)^{m} v_{1}+\cdots c_{n-2}\left(\frac{n-2}{n}\right)^{m} v_{n-1}+c_{n} v_{n}\right)=c_{n} v_{n}$.

So we only need to show that $c_{n}=(n+1) / 2$. Note that $\left(1, v_{n}\right)$ is also an eigenpair for $F^{\prime}$. So $\left\langle v_{n}, v_{k}\right\rangle=\left\langle F^{\prime} v_{n}, v_{k}\right\rangle=\left\langle v_{n}, F v_{k}\right\rangle=\frac{k}{n}\left\langle v_{n}, v_{k}\right\rangle$, and it implies that $\left\langle v_{n}, v_{k}\right\rangle=0$ for $k=0,1, \ldots, n-2$. Therefore $\left\langle v_{n}, x\right\rangle=$ $c_{0}\left\langle v_{n}, v_{0}\right\rangle+\cdots c_{n-2}\left\langle v_{n}, v_{n-2}\right\rangle+c_{n}\left\langle v_{n}, v_{n}\right\rangle=c_{n}\left\langle v_{n}, v_{n}\right\rangle$, and

$$
c_{n}=\frac{\left\langle x, v_{n}\right\rangle}{\left\langle v_{n}, v_{n}\right\rangle}=\frac{1+2+\cdots+n}{n}=\frac{n+1}{2} .
$$

It is interesting to note that the largest eigenvalue less than 1 is given by $\frac{n-2}{n}$ and so we expect the system to converge at the rate

$$
\left(\frac{n-2}{n}\right)^{n}
$$

Note that the explicit calculation for $F_{6}$ confirms this.

\section{Conclusion}

In this paper we have begun the process of using discrete time stochastic linear switching systems to study the mathematics of card shuffling. We have shown that any shuffling scheme can be represented by such a system. In future work we will extend this to other more complicated shuffles and will explore some of the tree based schemes proposed in [3] and [4]. 


\section{Appendix 1. Data sets}

(a) The top-in shuffle with 6 cards

\begin{tabular}{|cc|cc|}
\hline \hline 1 & 162 & 26 & 1 \\
2 & 141 & 27 & 2 \\
3 & 123 & 28 & 1 \\
4 & 97 & 29 & 1 \\
5 & 81 & 30 & 0 \\
6 & 54 & 31 & 0 \\
7 & 57 & 32 & 1 \\
8 & 58 & 33 & 1 \\
9 & 35 & 34 & 1 \\
10 & 27 & 35 & 1 \\
11 & 22 & 36 & 0 \\
12 & 23 & 37 & 1 \\
13 & 27 & 38 & 0 \\
14 & 13 & 39 & 0 \\
15 & 17 & 40 & 0 \\
16 & 15 & 41 & 0 \\
17 & 3 & 42 & 0 \\
18 & 11 & 43 & 0 \\
19 & 4 & 44 & 1 \\
20 & 3 & 45 & 0 \\
21 & 4 & 46 & 0 \\
22 & 1 & 47 & 0 \\
23 & 6 & 48 & \\
24 & 3 & 49 & \\
25 & 2 & $\ldots$ & $\ldots$ \\
\hline
\end{tabular}

(b) The top-in shuffle with 12 cards

\begin{tabular}{|rr|rr|rr|rr|rr|rr|}
\hline \hline 1 & 824 & 29 & 84 & 57 & 8 & 85 & 0 & 113 & 0 & 141 & 0 \\
2 & 748 & 30 & 83 & 58 & 7 & 86 & 0 & 114 & 0 & 142 & 0 \\
3 & 721 & 31 & 58 & 59 & 7 & 87 & 0 & 115 & 0 & 143 & 0 \\
4 & 634 & 32 & 47 & 60 & 7 & 88 & 0 & 116 & 0 & 144 & 0 \\
5 & 635 & 33 & 49 & 61 & 1 & 89 & 0 & 117 & 0 & 145 & 0 \\
6 & 501 & 34 & 41 & 62 & 6 & 90 & 0 & 118 & 0 & 146 & 0 \\
7 & 515 & 35 & 40 & 63 & 2 & 91 & 0 & 119 & 0 & 147 & 0 \\
8 & 465 & 36 & 45 & 64 & 1 & 92 & 0 & 120 & 0 & 148 & 0 \\
9 & 397 & 37 & 38 & 65 & 5 & 93 & 0 & 121 & 0 & 149 & 0 \\
10 & 386 & 38 & 33 & 66 & 7 & 94 & 0 & 122 & 0 & 150 & 0 \\
11 & 366 & 39 & 31 & 67 & 2 & 95 & 0 & 123 & 0 & $\ldots$ & $\ldots$ \\
12 & 325 & 40 & 21 & 68 & 3 & 96 & 0 & 124 & 0 & & \\
13 & 276 & 41 & 32 & 69 & 2 & 97 & 1 & 125 & 0 & & \\
14 & 246 & 42 & 19 & 70 & 4 & 98 & 0 & 126 & 0 & & \\
15 & 270 & 43 & 20 & 71 & 1 & 99 & 1 & 127 & 0 & & \\
16 & 233 & 44 & 15 & 72 & 1 & 100 & 0 & 128 & 0 & & \\
17 & 193 & 45 & 11 & 73 & 2 & 101 & 0 & 129 & 1 & & \\
18 & 164 & 46 & 21 & 74 & 3 & 102 & 0 & 130 & 0 & & \\
19 & 166 & 47 & 15 & 75 & 0 & 103 & 0 & 131 & 0 & & \\
20 & 166 & 48 & 10 & 76 & 0 & 104 & 0 & 132 & 0 & & \\
21 & 150 & 49 & 13 & 77 & 0 & 105 & 1 & 133 & 0 & & \\
22 & 145 & 50 & 15 & 78 & 0 & 106 & 1 & 134 & 0 & & \\
23 & 132 & 51 & 15 & 79 & 2 & 107 & 0 & 135 & 0 & & \\
24 & 113 & 52 & 10 & 80 & 1 & 108 & 0 & 136 & 0 & & \\
25 & 110 & 53 & 7 & 81 & 1 & 109 & 0 & 137 & 0 & & \\
26 & 82 & 54 & 6 & 82 & 2 & 110 & 0 & 138 & 0 & & \\
27 & 90 & 55 & 9 & 83 & 0 & 111 & 0 & 139 & 0 & & \\
28 & 72 & 56 & 7 & 84 & 0 & 112 & 0 & 140 & 0 & & \\
\hline & & & & & & & & & & \\
\hline
\end{tabular}


(c) The top-in shuffle with 54 cards

\begin{tabular}{|rr|rr|rr|rr|rr|rr|rr|}
\hline \hline 1 & 1773 & 29 & 1051 & 57 & 672 & 85 & 425 & 113 & 232 & 141 & 153 & 169 & 59 \\
2 & 1816 & 30 & 1025 & 58 & 679 & 86 & 388 & 114 & 225 & 142 & 128 & 170 & 75 \\
3 & 1889 & 31 & 1094 & 59 & 645 & 87 & 348 & 115 & 226 & 143 & 118 & 171 & 80 \\
4 & 1737 & 32 & 1052 & 60 & 607 & 88 & 355 & 116 & 218 & 144 & 131 & 172 & 85 \\
5 & 1775 & 33 & 954 & 61 & 606 & 89 & 359 & 117 & 226 & 145 & 122 & 173 & 66 \\
6 & 1712 & 34 & 957 & 62 & 576 & 90 & 365 & 118 & 220 & 146 & 124 & 174 & 85 \\
7 & 1610 & 35 & 933 & 63 & 557 & 91 & 353 & 119 & 201 & 147 & 140 & 175 & 76 \\
8 & 1599 & 36 & 961 & 64 & 575 & 92 & 335 & 120 & 196 & 148 & 116 & 176 & 78 \\
9 & 1613 & 37 & 964 & 65 & 588 & 93 & 334 & 121 & 190 & 149 & 132 & 177 & 56 \\
10 & 1668 & 38 & 968 & 66 & 572 & 94 & 308 & 122 & 191 & 150 & 118 & 178 & 71 \\
11 & 1584 & 39 & 873 & 67 & 563 & 95 & 313 & 123 & 170 & 151 & 118 & 179 & 72 \\
12 & 1515 & 40 & 913 & 68 & 533 & 96 & 284 & 124 & 192 & 152 & 124 & 180 & 71 \\
13 & 1501 & 41 & 845 & 69 & 494 & 97 & 324 & 125 & 191 & 153 & 97 & 181 & 69 \\
14 & 1493 & 42 & 842 & 70 & 458 & 98 & 326 & 126 & 197 & 154 & 113 & 182 & 71 \\
15 & 1404 & 43 & 854 & 71 & 499 & 99 & 248 & 127 & 154 & 155 & 107 & 183 & 71 \\
16 & 1370 & 44 & 807 & 72 & 474 & 100 & 290 & 128 & 186 & 156 & 94 & 184 & 72 \\
17 & 1412 & 45 & 833 & 73 & 487 & 101 & 259 & 129 & 161 & 157 & 124 & 185 & 67 \\
18 & 1372 & 46 & 731 & 74 & 500 & 102 & 279 & 130 & 163 & 158 & 104 & 186 & 56 \\
19 & 1402 & 47 & 820 & 75 & 462 & 103 & 255 & 131 & 149 & 159 & 105 & 187 & 74 \\
20 & 1265 & 48 & 727 & 76 & 450 & 104 & 272 & 132 & 174 & 160 & 85 & 188 & 41 \\
21 & 1280 & 49 & 752 & 77 & 397 & 105 & 261 & 133 & 181 & 161 & 120 & 189 & 45 \\
22 & 1170 & 50 & 715 & 78 & 427 & 106 & 259 & 134 & 145 & 162 & 98 & 190 & 55 \\
23 & 1260 & 51 & 686 & 79 & 409 & 107 & 229 & 135 & 144 & 163 & 89 & $\ldots$ & $\ldots$ \\
24 & 1179 & 52 & 694 & 80 & 409 & 108 & 243 & 136 & 175 & 164 & 74 & & \\
25 & 1232 & 53 & 731 & 81 & 454 & 109 & 263 & 137 & 139 & 165 & 83 & & \\
26 & 1132 & 54 & 675 & 82 & 392 & 110 & 238 & 138 & 159 & 166 & 90 & & \\
27 & 1162 & 55 & 668 & 83 & 401 & 111 & 229 & 139 & 124 & 167 & 97 & & \\
28 & 1166 & 56 & 667 & 84 & 387 & 112 & 220 & 140 & 144 & 168 & 85 & & \\
\hline
\end{tabular}

\section{Appendix 2. $\mathrm{C}++$ code for 6 cards}

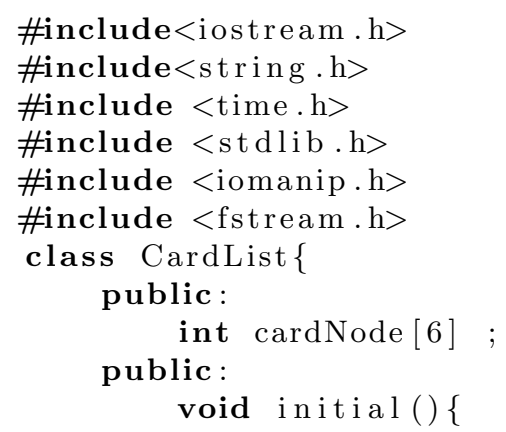




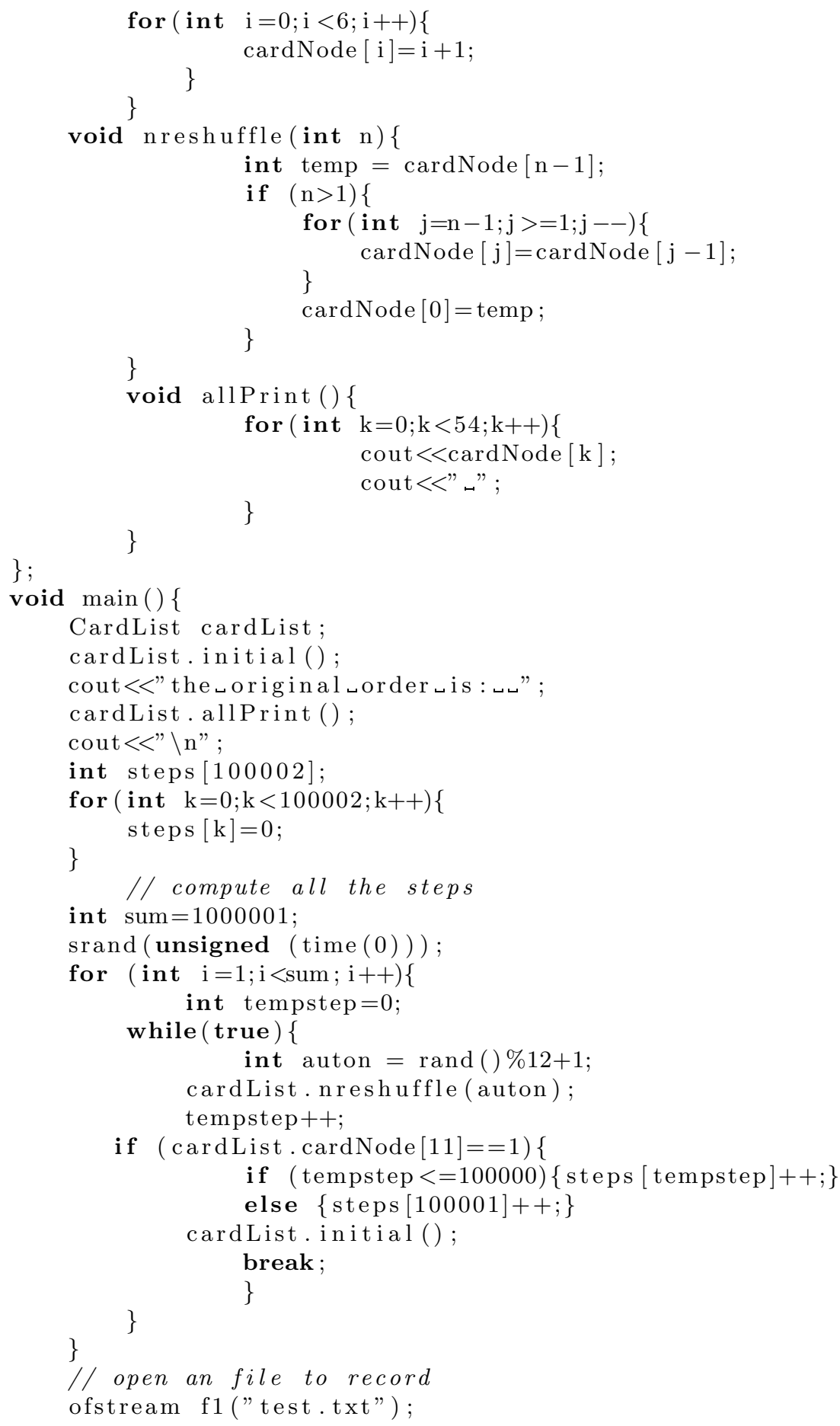




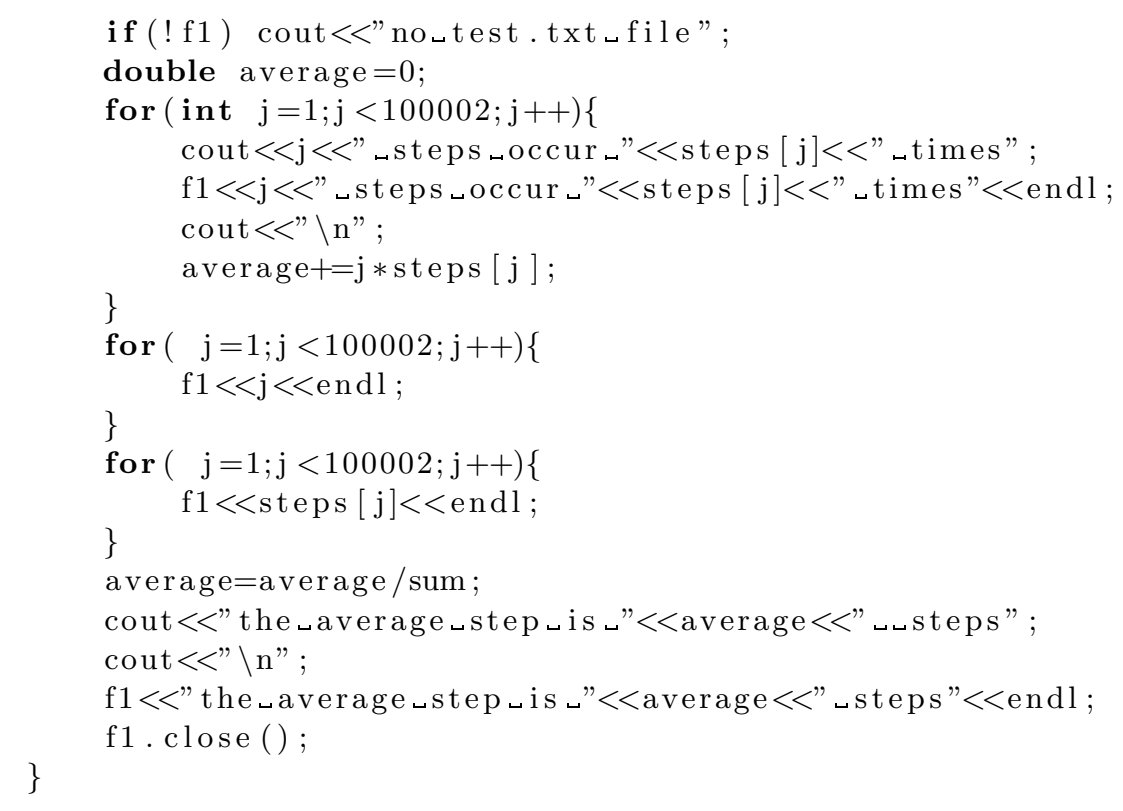

\section{References}

[1] D. Aldous and P. Diaconis, Strong uniform times and finite random walks, Advances in Applied Mathematics 8(1) (1987), 69-97. MR0876954

[2] P. Diaconis, Group representations in probability and statistics. Institute of Mathematical Statistics, Lecture Notes-Monograph Series, 11. Institute of Mathematical Statistics, Hayward, CA, 1988. MR0964069

[3] P. Diaconis and L. Saloff-Conte, Comparison techniques for random walk on finite groups, The annals of Probability 21 (1993), 2131-2156. MR1245303

[4] P. Diaconis and M. Shahshanani, Generating a random permutation with random transpositions, Z. Wahrsch. Verw. Gebiete 57 (1981), 159-179. MR0626813

[5] Y. Du and C. Martin, Switched systems as models for dynamic clinical trials, to appear, Communications in Information and Systems.

[6] B. Hanlon, V. Tyuryaev, and C. Martin, Stability of switched linear systems with Poisson switching, Communications in Information and Systems 11(4) (2011), 307-326. MR2950833 
[7] B. Hanlon, N. Wang, M. Egerstedt, and C. Martin, Switched linear systems: Stability and the convergence of random products, Communications in Information and Systems 11(4) (2011), 327-342. MR2950834

[8] L. Lovasz and P. Winkler, Mixing of random walks and other diffusions on a graph, Surveys in Combinatorics, 1995, P. Rowlinson ed., London Math. Soc. Lecture, Note Series, 218, 1995, pp. 119-154. MR1358634

[9] L. Lovasz and P. Winkler, Mixing times, Microsurveys in Discrete Probability, D. Aldous and J. Propp, eds, DIMACS Series in Discrete Math. and Theoretical Computer Science 41, Amer. Math. Soc., Providence RI, 1998, pp. 85-134. MR1630411

[10] Y. Pang, Random walk on a finite group, Master's Thesis, Texas Tech University, 2012.

Yulei PANG

Department of Mathematics and Statistics

Texas Tech University

USA

E-mail address: yulei.pang@ttu.edu

Alex Wang

Department of Mathematics and Statistics

TeXas Tech University

USA

E-mail address: alex.wang@ttu.edu

Xiaozhen Xue

Department of Computer Science

TeXas Tech University

USA

E-mail address: xiaozhen. xue@ttu.edu

Clyde F. Martin

Department of Mathematics and Statistics

Texas Tech University

USA

E-mail address: clyde.f.martin@ttu.edu

Received February 18, 2013 